\title{
Associação do sistema de histocompatibilidade humano com doenças of talmológicas
}

\section{Association of human histocompatibility antigens with ophthalmological disorders}

\author{
Crésio Alves ${ }^{1}$ \\ Isadora Meyer ${ }^{2}$ \\ Maria Betânia Pereira Toralles ${ }^{3}$ \\ Roberto Lorens Marback ${ }^{4}$
}

Esse trabalho foi parcialmente financiado pela FAPESB (Fundação de Amparo a Pesquisa do Estado da Bahia).

${ }^{1}$ Professor de Pediatria da Faculdade de Medicina da Universidade Federal da Bahia - UFBA - Salvador (BA) - Brasil.

${ }^{2}$ Acadêmica de Medicina da UFBA - Salvador (BA) Brasil.

${ }^{3}$ Professora de Genética da Faculdade de Medicina da UFBA - Salvador (BA) - Brasil.

${ }^{4}$ Professor Titular de Oftalmologia da Faculdade de Medicina da UFBA - Salvador (BA) - Brasil.

Endereço para correspondência: Crésio Alves. Rua Plínio Moscoso, 222 - Apto. 601 - Salvador (BA)

CEP 40157-190

E-mail: cresio.alves@uol.com.br

Recebido para publicação em 23.08.2005

Versão revisada recebida em 25.03.2006

Aprovação em 28.03.2006

\begin{tabular}{|l|}
\hline RESUMO \\
\hline Vários estudos têm procurado identificar marcadores genéticos para \\
doenças oftalmológicas. Dentre eles, destaca-se o antígeno de histo- \\
compatibilidade humano (Human Leukocyte Antigens). Situado no braço \\
curto do cromossomo 6, o sistema antígeno de histocompatibilidade \\
humano é conhecido por sua capacidade de conferir susceptibilidade ou \\
proteção a diferentes doenças. Em virtude do seu acentuado polimorfismo, \\
o tipo e a força da associação variam a depender da enfermidade e da raça \\
(etnia) estudadas. O surgimento de métodos moleculares para tipificação \\
dos alelos antígeno de histocompatibilidade humano e as recentes atua- \\
lizações de sua nomenclatura têm contribuído para o melhor entendimento \\
desse sistema. O presente trabalho tem por objetivos revisar a estrutura \\
e função do sistema antígeno de histocompatibilidade humano e relatar \\
suas associações com uveíte anterior aguda, penfigóide cicatricial ocular, \\
ceratocone de início na juventude e retinocoroidopatia "birdshot". \\
\hline
\end{tabular}

Descritores: Antígenos HLA; Complexo principal de histocompatibilidade; Retinite/ imunologia; Uveite/imunologia; Ceratocone/imunologia

\section{CONCEITO DO HLA}

O complexo principal de histocompatibilidade (MHC - Major Histocompatibility Complex) é responsável pela codificação das moléculas responsáveis pela apresentação de antígenos ao sistema imune. Em seres humanos, o MHC é denominado sistema HLA (Human Leukocyte Antigens). O lócus gênico para os genes que irão codificar estas proteínas está localizado no braço curto do cromossomo 6 (6p21.3). Neste lócus existem informações gênicas para codificar uma série de glicoproteínas envolvidas no processo de apresentação e reconhecimento de antígenos pelas células $\mathrm{T}^{(1-2)}$.

\section{CLASSES DO HLA}

Os genes do sistema HLA têm sido didaticamente agrupados em três regiões: classe I, II e III $^{(2-3)}$, sendo as duas primeiras referentes aos genes envolvidos com a resposta imune ${ }^{(4)}$. A região de classe I é composta pelos loci HLA-A, -B e -C, que codificam moléculas de histocompatibilidade presentes em quase todas as células somáticas do corpo humano ${ }^{(2-3)}$. A região de classe II é composta pelos loci HLA-DR, -DQ e -DP, que codificam as moléculas clássicas de histocompatibilidade, expressas em células imunes apresentadoras de antígenos (células B, células T ativadas, macrófagos, células dendríticas e células epiteliais tímicas) ${ }^{(2-4)}$. A apresentação de 
peptídeos antigênicos para os linfócitos T é a principal função das moléculas HLA. Os genes da classe III não expressam moléculas de histocompatibilidade, mas participam da resposta imune, codificando componentes do sistema complemento ${ }^{(2-4)}$.

Devido ao seu grande polimorfismo, os genes do sistema HLA apresentam frequiências alélicas variadas em diferentes populações ${ }^{(2)}$. Esta diversidade genética da espécie permite haver diferenças na susceptibilidade a doenças entre grupos geneticamente distintos, o que dificulta grandes epidemias ${ }^{(2,5)}$.

Essa revisão tem por objetivos rever a estrutura e função do sistema HLA, seus métodos de detecção, nomenclatura e relatar suas associações com uveíte anterior aguda, penfigóide cicatricial ocular, ceratocone de início na juventude e retinocoroidopatia "birdshot".

\section{DETECÇÃO DOS ANTÍGENOS E ALELOS HLA}

Os antígenos são detectados por testes imunológicos ${ }^{(2)}$. Para os de classe I utiliza-se testes sorológicos e para os de classe II, o método de microlinfocitotoxicidade celular mediada por anticorpo e dependente do complemento ${ }^{(6)}$ e o de cultura mista de linfócitos ${ }^{(3)}$. O primeiro tipifica antígenos HLA pela reação de um anticorpo contra as moléculas HLA presentes na superfície celular. No segundo, células com fenótipos conhecidos são utilizadas para definir as especificidades do HLA ${ }^{(2)}$. Os linfócitos $\mathrm{T}$ são utilizados para a tipificação de antígenos HLA-A, -B e -C, e os linfócitos B, para antígenos HLA-DR e -DQ. Não há anti-soros específicos para a tipificação de antígenos HLA-DP por este método ${ }^{(2)}$.

As técnicas de Biologia Molecular detectam não os antígenos HLA, mas os próprios alelos ou grupos de alelos polimórficos. Após a extração do DNA genômico, ele é amplificado por reação em cadeia da polimerase (polymerase chain reaction) - PCR e os alelos HLA são a genotipados através do método SSP (sequence specific primers) ou SSOP (sequence specific oligonucleotide probes) $)^{(3)}$.

\section{NOMENCLATURA DO SISTEMA HLA}

A nomenclatura do sistema HLA é determinada por um Comitê Internacional que se reúne periodicamente para revisar a padronização, conferindo novos nomes aos genes recém descobertos ou alterando a nomenclatura vigente ${ }^{(3)}$.

Os antígenos, definidos por métodos imunológicos, são representados pelo prefixo HLA seguidos por uma letra representando o locus gênico e por um ou dois algarismos representando o gene (e.g., HLA-A1, HLA-DR4, HLA-B2). Quando se faz referência ao locus $C$, incorpora-se a letra w (e.g., HLACw2, HLA-Cw3), para diferenciá-la do sistema complemento ${ }^{(3)}$.

Os alelos, definidos por métodos de Biologia Molecular, são representados pelo prefixo HLA, seguidos pela letra do locus, por um asterisco e por dois a oito dígitos (e.g., $H L A$ -
$D R B 1 * 1501,-D Q A 1 * 0102,-A * 0101)^{(7)}$. Os dois dígitos iniciais definem a família a qual pertence o alelo, correspondendo a sua família sorológica; o terceiro e o quarto são o código de variação, ou seja, especifica o alelo dentro da família; o quinto e o sexto dígitos descrevem variações daquele alelo; e o sétimo e oitavo descrevem variações nos íntrons (regiões 5' ou 3' do gene $)^{(7)}$.

Em alelos HLA de classe II dos loci -DQ e -DP, as cadeias polimórficas $\alpha$ e $\beta$ são designadas, respectivamente, pelas letras A e $B^{(2)}$. Como em alelos HLA-DR, o polimorfismo ocorre apenas nas cadeias $\beta$ (sua cadeia $\alpha$ não é polimórfica), acrescenta-se apenas letra $\mathrm{B}^{(3)}$. Os alelos HLA de classe I apresentam polimorfismo apenas na cadeia $\alpha$, não necessitando, portanto, dessa especificação.

Nos casos em que ocorre expressão nula (null) ou baixa (low) de um alelo, utiliza-se o sufixo opcional "N" ou "L". A revisão da nomenclatura realizada em 2004, no entanto, colocou como discutível a introdução desses sufixos adicionais, pois alguns casos em que se aplicou essa regra foram mais adiante considerados como indicativos de algum tipo de expressão protéica $^{(7)}$.

\section{ASSOCIAÇ̃̃O DO SISTEMA HLA COM DOENÇAS OFTALMOLÓGICAS}

As moléculas codificadas pelo sistema HLA são responsáveis pela apresentação dos antígenos, pois os linfócitos só reconhecem os antígenos quando eles estão ligados a moléculas $\mathrm{HLA}^{(8)}$. O conhecimento de como ocorre essa ligação é de fundamental importância para definir quais epítopos das células T participam do processo imunológico, facilitando, assim, o entendimento do mecanismo de associação do HLA com doenças intra-oculares causadas por linfócitos $\mathrm{T}$ que reconhecem e atacam antígenos próprios do olho ${ }^{(9-10)}$. O sistema HLA participa do controle da proteção e da susceptibilidade a doenças, influenciando o seu desenvolvimento e/ou funcionando como marcador da ativação da resposta imune ${ }^{(11)}$. No entanto, nem todos os indivíduos portadores de alelos associados à susceptibilidade a determinadas doenças irão desenvolvê-las; e nem todos os indivíduos acometidos por uma doença serão portadores dos genes HLA a ela associada ${ }^{(5)}$.

A forte associação de alguns genes HLA com determinadas doenças pode ser, na verdade, resultado do desequilíbrio de ligação desses genes com outros que estejam realmente associados à patologia ${ }^{(12)}$. A maior freqüência de alguns alelos pode ser secundária à sua inclusão em um haplótipo que abrange um alelo realmente associado à susceptibilidade à doença. Assim, tanto alelos como haplótipos podem funcionar como marcadores genéticos de doenças ${ }^{(13)}$.

O quadro 1 mostra os principais antígenos e alelos HLA associados a susceptibilidade a uveíte anterior aguda, penfigóide cicatricial ocular, ceratocone de início na juventude e retinocoroidopatia "birdshot". 


\begin{tabular}{|c|c|}
\hline \multicolumn{2}{|c|}{ Quadro 1. Associação do HLA com doenças oftalmológicas } \\
\hline Doença oftalmológica & $\begin{array}{l}\text { Antígenos/alelos do HLA } \\
\text { associados à susceptibilidade }\end{array}$ \\
\hline Uveíte anterior aguda & $\begin{array}{l}\text { HLA-B27, HLA-B51 } \\
\text { HLA-DQA } 1{ }^{*} 0101 \\
\text { HLA-DQB1*0501 } \\
\text { HLA-DRB1*0102. -DRB1*0404 }\end{array}$ \\
\hline Penfigóide cicatricial ocular & $\begin{array}{l}\text { HLA-A2, HLA-B8, HLA-B35, } \\
\text { HLA-B49, HLA-DQB1*0301 }\end{array}$ \\
\hline $\begin{array}{l}\text { Ceratocone de início } \\
\text { na juventude }\end{array}$ & HLA-A26, HLA-B40, HLA-DR9 \\
\hline Retinocoroidopatia "birdshot" & HLA-A29 \\
\hline
\end{tabular}

\section{MECANISMOS PATOGENÉTICOS}

Algumas hipóteses são sugeridas para explicar a participação dos genes HLA na patogênese de doenças. As principais delas são ${ }^{(3)}$ : (1) afinidade da molécula HLA pelo peptídeo causador de doença, levando os antígenos HLA a funcionarem como receptores para alguns agentes etiológicos, facilitando ou dificultando sua entrada na célula; (2) influência do HLA sobre a seleção tímica de linfócitos, determinando quais antígenos serão apresentados ao linfócito T; (3) mimetismo molecular entre antígenos de microorganismos infecciosos e antígenos do hospedeiro, suscitando um mecanismo de tolerância das células T contra esses patógenos, proporcionando, assim, susceptibilidade ou proteção contra doenças infecciosas; (4) expressão aberrante de moléculas HLA de classe II, isto é, células que normalmente não expressam moléculas HLA de classe II apresentando aos linfócitos $\mathrm{T}$ antígenos derivados da degradação do próprio tecido, causando, assim, doença auto-imune.

Em função da variabilidade genética observada em diferentes etnias, a combinação de alelos responsáveis pela manifestação de uma doença varia a depender da população estudada, embora alguns alelos prevaleçam independente da base étnica do grupo $^{(14)}$. Sendo assim, é recomendado que os grupos raciais sejam analisados individualmente para que suas particularidades possam ser percebidas.

\section{UVEÍTE ANTERIOR AGUDA}

A iridociclite aguda ou uveíte anterior aguda (UAA) consiste na inflamação da íris e do corpo ciliar ${ }^{(15)}$. Sua base genética tem sido estudada e a associação do antígeno HLA-B27, embora seja variável entre diferentes grupos étnicos, é considerada como elemento importante para o diagnóstico dessa inflamação ${ }^{(16-19)}$.

A uveíte anterior aguda associada ao antígeno HLA-B27 está associada a doenças sistêmicas, como espondilite anquilosante, artrite psoriática e doença inflamatória intestinal ${ }^{(11)}$. Já a uveíte em pacientes negativos para o antígeno HLA-B27 associa-se menos a doenças sistêmicas, pode ser crônica ou apresentar episódios recorrentes e geralmente é bilateral ${ }^{(11)}$.
Há controvérsia quanto à influência do HLA sobre o prognóstico. Alguns estudos referem que o antígeno HLA-B27 não influencia o prognóstico ${ }^{(11,20)}$, enquanto outros relatam que o prognóstico é melhor quando há positividade desse antígeno $^{(21)}$. Sua positividade recomenda a investigação de espondiloartropatias ${ }^{(16)}$. Além disso, existem evidências de maior susceptibilidade a uveíte anterior aguda secundária a infecção aguda ocular por Bartonella henselae em portadores do antígeno HLA-B2 $7^{(17,22)}$.

Alguns estudos propõem a associação da uveíte da doença de Behçet com o antígeno HLA-B51 ${ }^{(23-24)}$. A síndrome constituída por nefrite tubulointersticial e uveíte crônica ocorre com maior freqüência em pacientes portadores dos alelos $H L A$ $D Q A 1 * 0101,-D Q B 1 * 0501$ e $-D R B 1 * 0102$, do antígeno HLAB14 e do haplótipo HLA-DQA1*01-DQB1*05-DRB1*01. Outras uveítes crônicas associadas a moléculas HLA de classe II são a doença de Vogt-Koyanagi-Harada (VKH) e a uveíte associada à artrite reumatóide juvenil ${ }^{(25)}$. A primeira associa-se aos alelos $H L A-D R B 1 * 0404$ e $-D R B 1 * 0407^{(25)}$ e a segunda, ao antígeno HLA-B27(26).

Recentemente, uma terapia de indução de tolerância utilizando o peptídeo HLA-B27PD, derivado de antígenos HLA da própria retina dos pacientes, mostrou eficácia na melhora da uveíte auto-imune ${ }^{(27)}$. Trata-se de um peptídeo derivado de uma sequiência comum a muitas moléculas HLA-B (incluindo os antígenos HLA-B27 e -B51) associadas com a uveíte que apresentam homologia seqüencial com o epítopo do antígeno $\mathrm{S}$ (PDS) da retina ${ }^{(23,28)}$. Os mecanismos imunológicos de tolerância ainda não foram completamente esclarecidos, mas supõe-se que, através do reconhecimento do epítopo citado, ocorra geração de células $\mathrm{T}$ supressoras e alterações na regulação de algumas citocinas ${ }^{(29)}$.

A positividade do antígeno HLA-B27 também parece implicar em maiores níveis de TNF- $\alpha$ (citocina que participa ativamente na patogênese da uveíte) no humor aquoso de pacientes com uveíte ativa ${ }^{(30)}$. Novas drogas inibidoras do TNF- $\alpha$ têm sido propostas para o tratamento da uveíte associada ao antígeno HLA-B27 $7^{(31)}$.

Heiligenhaus et al. (2004), encontraram associação da uveíte anterior com antígenos HLA-DR, porém somente em pacientes negativos para o HLA-B2 $7^{(11)}$. Dentre aqueles positivos para antígenos HLA-DR e negativos para-B27, os níveis plasmáticos de antígenos HLA-DR eram mais elevados nos com curso crônico da doença do que nos com curso agudo.

\section{PENFIGÓIDE CICATRICIAL OCULAR}

Penfigóide cicatricial ocular (PCO), variante do pênfigo membrano-mucoso $(\mathrm{PMM})^{(32)}$, é uma doença auto-imune que forma bolhas e cicatrizes em conjuntiva, podendo causar perda da visão quando inadequadamente tratada ${ }^{(13)}$.

Em caucasianos, os haplótipos $H L A-D R 4-D Q w 7$ e -DR5$D Q w 7^{(13)}$, especialmente o alelo $H L A-D Q B 1^{*} 0301^{(13,33-36)}$, são tidos como marcadores genéticos para essa doença. Segundo 
Ahmed et al. (1991), o alelo em questão favorece a susceptibilidade ao PCO tanto em homozigose quanto em heterozigose, mostrando caráter dominante na influência sobre a doença ${ }^{(13)}$. Chan et al. (1997), encontraram diferenças significantes entre as frequiências do alelo $H L A-D Q B 1 * 0301$ em controles e pacientes portadores de $\mathrm{PCO}$ em combinação com diversas variantes do PMM (formas mucosa, mucosa-oral ou cutânea) ${ }^{(37)}$.

É possível que a resposta auto-imune desses pacientes envolva o reconhecimento por linfócitos $\mathrm{T}$ de moléculas expressas pelo alelo $H L A-D Q B 1 * 0301$ ou por outros alelos HLA de classe II em desequilíbrio de ligação com o primeiro, as quais tenham afinidade com a membrana basal da conjuntiva facilitando a produção de anticorpos contra $\operatorname{estas}^{(13,38)}$. Apesar das evidentes associações do PCO com o alelo HLA$D Q B 1 * 0301$, estudo realizado com gêmeos, dentre os quais apenas um era afetado pela doença, sugere que os fatores genéticos não são suficientes para o seu desenvolvimento, havendo também a participação de fatores ambientais ${ }^{(39)}$. Significativo número de pacientes são portadores dessa doença mesmo sem possuir esse alelo, logo, é possível que ele apareça como marcador genético por estar em desequilíbrio de ligação com outros genes associados à susceptibilidade ao $\mathrm{PCO}^{(1)}$.

O HLA de classe I não parece estar associado à doença ${ }^{(1)}$, embora Mondino et al. ${ }^{(40-42)}$ tenham sugerido associação com o antígeno HLA-B12. Em 1989, foi sugerida a associação com os antígenos HLA-A2, -B8, -B35 e -B49 ${ }^{(43)}$.

\section{CERATOCONE DE INÍCIO NA JUVENTUDE}

O ceratocone, ectasia corneana não inflamatória e autolimitada, consiste em afinamento e protusão da porção central ou para-central da córnea, que acaba por adquirir forma côni$\mathrm{ca}^{(44)}$. Suspeita-se que possua caráter hereditário. O gene VISX1 foi identificado e há outros loci sendo investigados. A apoptose de ceratócitos, que ocorre com frequiência muito maior em córneas com ceratocone do que em córneas normais consiste em um reforço à possível influência genética sobre a doença ${ }^{(44)}$. O antígeno HLA-B7 é sugerido como marcador de proteção ${ }^{(45)}$. Ihalainen (1986) realizou a tipagem de antígenos HLA-A, -B e -C em famílias com múltiplos casos de ceratocone $^{(46)}$. Em 15 famílias com mais de um filho afetado, 27 dentre 30 crianças com ceratocone possuíam haplótipos HLA semelhantes aos dos pais também afetados, sugerindo que, dentro de uma família multi-afetada, o haplótipo HLA pode funcionar como um marcador da doença. Em japoneses que desenvolveram ceratocone antes dos 20 anos, a frequiência dos antígenos HLA-A26, -B40 e -DR9 parece ser maior do que naqueles que desenvolveram-na com idade superior a esta ${ }^{(47)}$.

\section{RETINOCOROIDOPATIA "BIRDSHOT"}

A retinocoroidopatia "birdshot" (RBS) é um tipo raro de uveíte inflamatória posterior, bilateral, crônica e auto-imune.
Caracteriza-se por pontos hipopigmentados difusos em epitélio pigmentar e coróide, vitreíte e vasculopatia retiniana, levando a edema macular cistóide e papiledema ${ }^{(48)}$. Sua patogênese é desconhecida, mas há forte associação com o antígeno HLA-A29 ${ }^{(9,49-50)}$. Esse antígeno, presente em $80-98 \%$ dos pacientes, aumenta significativamente o risco para RBS e sua positividade serve como diagnóstico diferencial com outras enfermidades de acometimentos oftalmológicos semelhantes, como sífilis, tuberculose e sarcoidose $\mathrm{e}^{(50-51)}$. A aparência amarelada do fundo do olho e a presença do antígeno HLA-A29 indicam o diagnóstico da patologia ${ }^{(52)}$. Existem dois subtipos de antígenos HLA-A29 (-A29.1 e -A29.2), sendo que o segundo é o predominante em pacientes com RBS $^{(53-54)}$. Não se sabe, no entanto, se sua maior prevalência se deva a uma real associação com a doença ou se ocorre pelo fato de ele ser mais comum na população geral ${ }^{(50)}$. Especula-se que o antígeno HLA-A29.1 seja uma mutação do -A29.2 e que essa mutação confira resistência à RBS, inibindo a interação dessas moléculas com linfócitos $\mathrm{T}$ e conseqüente ativação dessas células ${ }^{(50)}$. Mas isso ainda é controverso, sendo que alguns estudos sugerem que ambos os antígenos estão igualmente associados à susceptibilidade ${ }^{(9)}$.

Boisgérault et al. (1996) sugeriram que antígenos próprios da retina dos pacientes, com afinidade pelas moléculas HLAA29, funcionariam como auto-antígenos, implicando em RBS auto-imune ${ }^{(9)}$. Suspeita-se também da participação de agentes infecciosos na patogênese que ao inserir seus epítopos em moléculas HLA-A29 do hospedeiro, facilitariam a apresentação dessas moléculas próprias do organismo aos linfócitos $\mathrm{T}$ citotóxicos, gerando doença auto-imune ${ }^{(50)}$.

Uma síndrome associada ao antígeno HLA-A29 foi sugerida por Bloch-Michel, Frau, (1991), englobando a RBS e outras condições cuja expressão pode ser geneticamente determinada, como envolvimento do pólo posterior do globo ocular, curso severo da doença e prognóstico ruim, apesar da terapia com corticóide ${ }^{(53)}$. Em contraste, os pacientes com vasculite retiniana idiopática negativos para esse antígeno apresentaram envolvimento ocular mais periférico, curso menos severo da doença e melhor prognóstico ${ }^{(53)}$.

Dessa forma, a análise do HLA dos pacientes com suspeita de RBS é fundamental para a detecção e implantação precoce do tratamento, essenciais para preservação da acuidade visual ${ }^{(55)}$.

\section{CONCLUSÕES}

A associação de algumas doenças oftalmológicas com o sistema HLA destaca o caráter genético e a participação do sistema imune na patogênese dessas doenças. Entretanto, a presença de um alelo ou haplótipo associado à susceptibilidade não é suficiente para o desenvolvimento da doença. Isso reforça a noção de que além do sistema HLA outros fatores contribuem com a patogênese dessas doenças.

Devido ao grande polimorfismo do sistema HLA, suas frequiências alélicas são bastante variadas em diferentes po- 
pulações, de forma que os marcadores estabelecidos para um determinado grupo étnico não são necessariamente os mesmos de outro. Assim, estudos em populações etnicamente distintas, especialmente em populações muito miscigenadas como a brasileira, são recomendados para verificar se a susceptibilidade e a proteção se associam aos alelos descritos nas populações já investigadas, predominantemente, caucasiana ou asiática.

A melhor compreensão do papel das moléculas HLA em doenças oftalmológicas irá proporcionar informações a respeito de sua patogênese, principalmente sobre os aspectos autoimunes, contribuindo para a formação de estratégias preventivas e para a instituição de terapias mais seguras. A análise do HLA de pacientes com suspeita dessas doenças é fundamental para o diagnóstico e o tratamento precoces, beneficiando a preservação da acuidade visual desses pacientes.

\section{ABSTRACT}

Many studies have been trying to identify genetic markers for opthalmological diseases, including, among others, the HLA (Human Leukocyte Antigens). Localized on the short arm of chromosome 6, the human leukocyte antigen system is well known for its capacity to confer susceptibility or resistance to different diseases. In view of its accentuated polymorphism, the strength and type of association differs with the disease and sometimes, with the studied ethnic-racial group. The development of molecular methods to typify HLA alleles and recent updates of their nomenclature has contributed to a better understanding of this system. In this review, some aspects of the human leukocyte antigen system are discussed, such as the methods of detection, nomenclature and association with acute anterior uveitis, ocular cicatricial pemphigoid, young-onset keratoconus and birdshot retinochoroidopathy.

Keywords: HLA antigens; Major histocompatibility complex; Retinitis/immunology; Uveitis/immunology; Keratoconus/immunology

\section{REFERÊNCIAS}

1. Setterfield J, Theron J, Vaughan RW, Welsh KI, Mallon E, Wojnarowska F, et al. Mucous membrane pemphigoid: HLA-DQB $1 * 0301$ is associated with all clinical sites of involvement and may be linked to antibasement membrane $\mathrm{IgG}$ production. Br J Dermatol. 2001;145(3):406-14.

2. Alves C, Souza T, Veiga S, Alves CO, Toralles MB, Lemaire D. A importância do sistema de histocompatibilidade humano (HLA) em Pediatria. Pediatria (São Paulo). 2005;27(4):274-86.

3. Donadi EA. Como entender a nomenclatura e os mecanismos de associação entre os antígenos e os alelos de histocompatibilidade com as doenças. Medicina (Ribeirão Preto). 2000;33:7-18.

4. Klein J, Sato A. The HLA System. First of two parts. N Engl J Med. 2000; 343(10):702-9.

5. Van Rood JJ. The impact of the HLA-system in clinical medicine. Shweiz Med Wschr. 1993;123(3):85-92.

6. Terasaki PI, McClelland JD. Microdroplet assay of human serum cytotoxins. Nature. 1964;204:998-1000.

7. Marsh SGE, Ekkehard AD, Bodmer WF, Bontrop RE, Dupont B, Erlich HA, et al. Nomenclature for factors of the HLA system (2004). Tissue Antigens. 2005;65(4):301-69.

8. Probst CM, Bompeixe EP, Pereira NF, de O Dalalio MM, Visentainer JE, Tsuneto LT, et al. HLA polymorphism and evaluation of European, African, and Amerindian contribution to the white and mulatto populations from Paraná, Brazil. Hum Biol. 2000;72(4):597-617.

9. Boisgerault F, Khalil I, Tieng V, Connan F, Tabary T, Cohen JH, et al. Definition of the HLA-A29 peptide ligand motif allows prediction of potential T-cell epitopes from the retinal soluble antigen, a candidate autoantigen in birdshot retinopathy. Proc Natl Acad Sci USA. 1996;93(8):3466-70.

10. Fernandez-Melon J, Munoz-Fernandez S, Hidalgo V, Bonilla-Hernan G, Schilincker A, Fonseca A, et al. Uveitis as the initial clinical manifestation in patients with spondyloarthropathies. J Rheumatol. 2004;31(3):524-7.

11. Heiligenhaus A, Rebmann V, Neubert A, Plewa S, Ferencik S, Vogeler U, et al. Soluble HLA class I and HLA-DR plasma levels in patients with anterior uveitis. Tissue Antigens. 2004;63(4):369-75.

12. Levinson RD, Park MS, Rikkers SM, Reed EF, Smith JR, Martin TM, et al. Strong associations between specific HLA-DQ and HLA-DR alleles and the tubulointerstitial nephritis and uveitis syndrome. Invest Ophthalmol Vis Sci. 2003;44(2):653-7.

13. Ahmed AR, Foster S, Zaltas M, Notani G, Awdeh Z, Alper CA, et al. Association of DQw7 (DQB1*0301) with ocular cicatricial pemphigoid. Proc Natl Acad Sci USA. 1991;88(24):11579-82.

14. Zhang X, Wei S, Yang S, Wang Z, Zhang A, He P, et al. HLA-DQA1 and DQB1 alleles are associated with genetic susceptibility to psoriasis vulgaris in Chinese Han. Int J Dermatol. 2004;43(3):181-7.

15. Wakefield D, Montanaro A, McCluskey P. Acute anterior uveitis and HLAB27. Surv Ophtalmol. 1991;36(3):223-32.

16. Huhtinen M, Karma A. HLA-B27 typing in the categorization of uveitis in a HLA-B27 rich population. Br J Ophthalmol. 2000;84(4):413-6.

17. Kerkhoff FT, Rothova A. Bartonella henselae associated uveitis and HLA-B27. Br J Ophthalmol. 2000;84(10):1125-9.

18. Smith JR. HLA-B27 associated uveitis. Ophthalmol Clin North Am. 2002;15 (3):297-307.

19. Martin TM, Kurz DE, Rosenbaum JT. Genetics of uveitis. Ophthalmol Clin North Am. 2003;16(4):555-65.

20. Lin JY, Lin SL, Chen WL, Chen YS. HLA-B27-associated uveitis with a chorioretinitis manifestation. Ophthalmic Surg Lasers Imaging. 2005;36(2):158-62.

21. Monnet D, Breban M, Hudry C, Dougados M, Brezin AP. Ophthalmic findings and frequency of extraocular manifestations in patients with HLA-B27 uveitis: a study of 175 cases. Ophthalmology. 2004;111(4):802-9.

22. Drancourt M, Bodaghi B, Lepidi H, Le Hoang P, Raoult D. Intraocular detection of Bartonella henselae in a patient with HLA-B27 uveitis. J Clin Microbiol. 2004;42(4):1822-5.

23. Gül A, Uyar FA, Inanc M, Öcal L, Tugal-Tutkun I, Aral O, et al. Lack of association of HLA-B*51 with a severe disease course in Behçet's disease. Rheumatology (Oxford). 2001;40(6):668-72.

24. Mizuki N, Ota M, Katsuyama Y, Yabuki K, Ando H, Shiina T, et al. Sequencing-based typing of HLA-B*51 alleles and the significant association of HLAB*5101 and -B*5108 with Behcet's disease in Greek patients. Tissue Antigens. 2002;59(2):118-21.

25. Levinson RD, See RF, Rajalingam R, Reed EF, Park MS, Rao NA, et al. HLADRB1 and -DQB1 alleles in mestizo patients with Vogt-Koyanagi-Harada's disease in Southern California. Hum Immunol. 2004;65(12):1477-82.

26. Bosch-Driessen EH, Lardy NM, Rothova A. Antinuclear antibody and HLA-B27 positive uveitis: combination of two diseases? Br J Ophthalmol. 1997;81(9):771-3.

27. Thurau SR, Fricke H, Burchardi C, Diedrichs-Mohring M, Wildner G. Longterm follow-up of oral tolerance induction with HLA-peptide B27PD in patients with uveitis. Ann N Y Acad Sci. 2004;1029:408-12.

28. Thurau SR, Diedrichs-Mohring M, Fricke H, Arbogast S, Wildner G. Molecular mimicry as a therapeutic approach for an autoimmune disease: oral treatment of uveitis-patients with an MHC-peptide crossreactive with autoantigen-first results. Immunol Lett. 1997;57(1-3):193-201.

29. Thurau SR, Wildner G. Oral tolerance for treating uveitis - new hope for an old immunological mechanism. Prog Retin Eye Res. 2002;21(6):577-89.

30. Perez-Guijo V, Santos-Lacomba M, Sanchez-Hernandez M, Castro-Villegas MC, Gallardo-Galera JM, Collantes-Estevez E. Tumour necrosis factor-alpha levels in aqueous humour and serum from patients with uveitis: the involvement of HLAB27. Curr Med Res Opin. 2004;20(2):155-7.

31. Suhler EB, Martin TM, Rosenbaum JT. HLA-B27-associated uveitis: overview and current perspectives. Curr Opin Ophthalmol. 2003;14(6):378-83.

32. Carrozzo M, Fasano ME, Broccoletti R, Carbone M, Cozzani E, Rendine S, et al. HLA-DQB1 alleles in Italian patients with mucous membrane pemphigoid predominantly affecting the oral cavity. Br J Dermatol. 2001;145(5):805-8. 
33. Haider N, Neuman R, Foster CS, Ahmed AR. Report on the sequence of DQB1*0301 gene in ocular cicatricial pemphigoid patients. Curr Eye Res. 1992;11(12):1233-8.

34. Chan LS, Wang T, Wang XS, Hammerberg C, Cooper KD. High frequency of HLA-DQB $1 * 0301$ allele in patients with pure ocular cicatricial pemphigoid. Dermatology. 1994;189(1):99-101.

35. Yunis JJ, Mobini N, Yunis EJ, Alper CA, Deulofeut R, Rodriguez A, et al. Common major histocompatibility complex class II markers in clinical variants of cicatricial pemphigoid. Proc Natl Acad Sci USA. 1994;91(16):7747-51.

36. Drouet M, Delpuget-Bertin N, Vaillant L, Chauchaix S, Boulanger MD, Bonnetblanc JM, et al. HLA-DRB1 and HLA-DQB1 genes in susceptibility and resistance to cicatricial pemphigoid in French Caucasians. Eur J Dermatol. 1998;8(5):330-3.

37. Chan LS, Hammerberg C, Cooper KD. Significantly increased occurrence of HLA-DQB $1 * 0301$ allele in patients with ocular cicatricial pemphigoid. J Invest Dermatol. 1997;108(2):129-32.

38. Delgado JC, Turbay D, Yunis EJ, Yunis JJ, Morton ED, Bhol K, et al. A common major histocompatibility complex class II allele HLA-DQB1* 0301 is present in clinical variants of pemphigoid. Proc Natl Acad Sci USA. 1996; 93(16):8569-71.

39. Bhol K, Udell I, Haider N, Yunis JJ, Mohimen A, Neuman R, et al. Ocular cicatricial pemphigoid. A case report of monozygotic twins discordant for the disease. Arch Ophthalmol. 1995;113(2):202-7.

40. Mondino BJ, Brown SI, Rabin BS. HLA antigens in ocular cicatricial pemphigoid. Br J Ophthalmol. 1978;62(4):265-7.

41. Mondino BJ, Brown SI, Rabin BS. Autoimmune phenomena of the external eye. Ophthalmology. 1978;85(8):801-17.

42. Mondino BJ, Brown SI, Rabin BS. HLA antigens in ocular cicatricial pemphigoid. Arch Ophthalmol. 1979;97(3):479.

43. Zaltas MM, Ahmed R, Foster CS. Association of HLA-DR4 with ocular cicatricial pemphigoid. Curr Eye Res. 1989;8(2):189-93.
44. Diniz CM, Tzelikis PF, Rodrigues Junior A, Alvim H da S, Dantas RR, Figueredo AR. [Unilateral keratoconus associated with continual eye rubbing due to nasolacrimal obstruction: case report]. Arq Bras Oftalmol. 2005;68(1):122-5. Portuguese.

45. Harrison RJ, Klouda PT, Easty DL, Manku M, Charles J, Stewart CM. Association between keratoconus and atopy. Br J Ophthalmol. 1989;73(10):816-22.

46. Ihalainen A. Clinical and epidemiological features of keratoconus genetic and external factors in the pathogenesis of the disease. Acta Ophthalmol Suppl. 1986;178:1-64.

47. Adachi W, Mitsuishi Y, Terai K, Nakayama C, Hyakutake Y, Yokoyama J, et al. The association of HLA with young-onset keratoconus in Japan. Am J Ophthalmol. 2002;133(4):557-9.

48. Baarsma GS, Priem HA, Kijlstra A. Association of birdshot retinochoroidopathy and HLA-A29 antigen. Curr Eye Res. 1990;9 Suppl:63-8.

49. Nussenblatt RB, Mittal KK, Ryan S, Green WR, Maumenee AE. Birdshot retinochoroidopathy associated with HLA-A29 antigen and immune responsiveness to retinal S-antigen. Am J Ophthalmol. 1982;94(2):147-58.

50. Gasch AT, Smith JA, Whitcup SM. Birdshot retinochoroidopathy. Br J Ophthalmol. 1999;83(2):241-9.

51. Vianna RNG, Serop S, Priem HA. Coriorretinopatia tipo Birdshot atípica. Rev Bras Oftalmol. 1994;53(5):77-81.

52. Gaudio PA, Kaye DB, Crawford JB. Histopathology of birdshot retinochoroidopathy. Br J Ophthalmol. 2002;86(12):1439-41. Comment in: Br J Ophthalmol. 2002;86(12):1324-5.

53. Bloch-Michel E, Frau E. Birdshot retinochoroidopathy and HLA-A29+ and HLA-A29- idiopathic retinal vasculitis: comparative study of 56 cases. Can J Ophthalmol. 1991;26(7):361-6.

54. LeHoang P, Ozdemir N, Benhamou A, Tabary T, Edelson C, Betuel H, et al HLA-A29.2 subtype associated with birdshot retinochoroidopathy. Am J Ophthalmol. 1992;113(1):33-5.

55. Abrantes P, Martins M, Ferreira P, Ganhão F. [Birdshot retinochoroidopathy. Five clinical cases analysis]. Arch Soc Esp Oftalmol. 2002;77(5):247-55. Spanish.

ORGANIZAÇÃO:

Sociedade Brasileira de Visão Subnormal

INFORMAÇÕES:

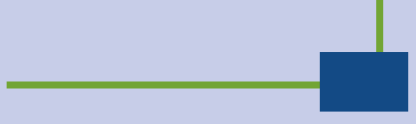

Tel.: (11) 3266-4000 com Fabrício

Home-page: www.visaosubnormal.org.br 